\title{
Estado nutricional de niños y niñas indígenas de hasta seis años de edad en el resguardo Embera-Katío, Tierralta, Córdoba, Colombia
}

\author{
Berta Nelly Restrepo ${ }^{1}$, María Teresa Restrepo ${ }^{2}$, Juan Camilo Beltrán ${ }^{\text {, }}$ Mónica Rodríguez ${ }^{\text {, }}$ \\ Ruth Emilia Ramírez ${ }^{1}$ \\ 1 Instituto Colombiano de Medicina Tropical-Instituto de Ciencias de la Salud-CES, Sabaneta, Antioquia, \\ Colombia. \\ ${ }^{2}$ Grupo de Investigación en Alimentación y Nutrición Humana, Escuela de Nutrición y Dietética, Universidad \\ de Antioquia, Medellín, Colombia.
}

Introducción. El conocimiento del estado nutricional de la población infantil es un valioso indicador del desarrollo de una población.

Objetivo. Determinar la prevalencia de desnutrición en niños y niñas indígenas Embera-Katío de hasta seis años de edad e identificar algunos de sus factores condicionantes.

Materiales y métodos. Se realizó un estudio transversal en el resguardo Embera-Katío, municipio de Tierralta, departamento de Córdoba, Colombia, en 272 niños de hasta seis años de edad a quienes se les midió el peso, la estatura y el perímetro cefálico. En 194 hogares se realizó una encuesta sobre aspectos alimentarios y de salud y a 172 niños y niñas se les practicó un examen de materia fecal.

Resultados. La prevalencia de desnutrición crónica moderada y grave fue de $63,6 \%$ y de desnutrición aguda leve y moderada fue de $8,8 \%$. La desnutrición crónica se asoció con el incremento de la edad $(p<0,005)$ y fue mayor en hombres que en mujeres $(89,9$ vs. $80,4 \%: p$ $=0,028$ ). Se observó mayor prevalencia de desnutrición crónica en los niños con infección respiratoria aguda, con enfermedad diarreica aguda y en hogares con más de tres niños y niñas menores de cinco años. En $74 \%$ de los niños y niñas, el perímetro cefálico fue inferior al percentil 5. La alimentación de los niños y niñas después del destete fue básicamente arroz y maíz $(92,8 \%)$ y plátano (80,9\%). Sólo $28,9 \%$ consumió carne, $4,6 \%$ huevos y $4,1 \%$ leche. La prevalencia de parasitosis intestinal fue de $78,1 \%$.

Conclusiones. La prevalencia de desnutrición crónica en los niños y niñas Embera-Katio fue elevada, lo cual es el reflejo de las condiciones adversas a las que están sometidos.

Palabras clave: salud de la población indígena, preescolar, niño, estado nutricional, desnutrición, morbilidad.

Nutritional status of indigenous children aged up to six years in the Embera-Katio Indian reserve in Tierralta, Cordoba, Colombia

Introduction. Knowledge of the nutritional state of the children is an valuable indicator of the general state of development in an entire population.

Objective. The prevalence of malnutrition was determined in Embera-Katio's indigenous children, aged 0-6 years, and associated factors associated with malnutrition were identified. Materials and methods. A cross-sectional study was undertaken in the Resguardo EmberaKatío, in Tierralta in the Province of Cordoba, located in northern Colombia. The weight, height and cephalic perimeter was assessed for each of 272 children under six years of age. Nutritional paramterers were surveyed in194 homes; fecal samples of 172 children were examined for evidence of intestinal parasites.

Results. Prevalence of moderate and severe chronic malnutrition was found in $63.6 \%$ of the children's; $8.8 \%$ were categorized with slight and moderate acute malnutrition. Chronic malnutrition was associated with age increase $(p=0.005)$ and was greater in males than in females $(89.9 \%$ versus $80.4 \% ; p=0.028)$. Chronic malnutrition was greater in children with 
acute respiratory infection, acute diarrheas, and in homes with three or more children below the age of five. In $74 \%$ of the children, the cephalic perimeter was below the fifth percentile. After weaning, the children were fed a basic diet of rice and corn $(80.4 \%)$ and plantain $(80.9 \%)$. Only $28.9 \%$ ate meat, $4.6 \%$ eggs, $4.1 \%$ milk and $1.5 \%$ fruits and vegetables. The prevalence of intestinal parasitism was $78.1 \%$.

Conclusions. The prevalence of chronic malnutrition in the Embera-Katio children was high, reflecting the adverse nutritional and economic conditions in which they live.

Key words: Indigenous health; child, preschool; child, nutritional status, morbidity, foods.

El estado nutricional de una comunidad tiene estrecha asociación, entre otros, con factores socioeconómicos de la sociedad a la que pertenece. La pobreza hace vulnerables social y biológicamente a la familia y al individuo; una de sus consecuencias biológicas es la baja estatura de la población infantil $(1,3)$. Las condiciones de pobreza en que viven los indígenas les niegan la posibilidad de tener una calidad de vida adecuada. En un estudio sobre salud y supervivencia de indígenas Mayas que vivían en condiciones de pobreza, Mata (1) concluyó que, "Las pobres condiciones socioeconómicas fueron determinantes y predominantes en las deficiencias biológicas y ambientales de la población indígena y su ecosistema".

Según la reunión de Pueblos Indígenas y Salud, cerca de 43 millones de indígenas de la región de las Américas tienen condiciones de vida y salud deficientes (4). Para Mudarra, la pobreza que padecen los sectores indígenas de Panamá es uno de los factores que contribuye enormemente a que ese sector posea el más alto índice de desnutrición: el $94 \%$ de los indígenas es pobre y de éste, el $87,7 \%$ padece de pobreza extrema (5). Entre la población indígena de Colombia se presenta una de las más altas tasas de mortalidad infantil del mundo (6). Un promedio de 250 de cada mil niños en las comunidades Paeces en el Cauca, Awá Kwaikeres en Nariño y Emberá en Antioquia fallecen antes de los seis años de edad (6). La principal causa de mortalidad infantil es la

\footnotetext{
Correspondencia:

Berta Nelly Restrepo, Instituto Colombiano de Medicina Tropical-CES, Carrera 43른 52 sur-99 Sabaneta, Antioquia, Colombia. Teléfono: (574) 3053500, fax: (574) 3014258. brestrepo@ces.edu.co

Recibido: 25/04/06; aceptado: 29/09/06
}

desnutrición que hace a los niños mucho más vulnerables a cualquier tipo de enfermedad (6). Los problemas de salud pública y el deterioro de la calidad de vida de las comunidades indígenas están estrechamente ligados con los procesos de cambio que se viven en las regiones, manifestados por diferentes actividades económicas y sociales que impactan directa o indirectamente el medio ambiente y la salud de esta población. La instauración en zonas indígenas de grandes proyectos de explotación petrolera, el desarrollo vial o hidroeléctrico y los cultivos ilícitos, entre otros, generan diversos problemas socioeconómicos y de salud (7). En la zona noroeste de Colombia, al sur del departamento de Córdoba, en el municipio de Tierralta, habitan los indígenas Embera-Katio del Alto Sinú en un resguardo de 103.517 hectáreas dentro del Parque Natural Paramillo (Alianza Indígena de cabildos menores río Esmeralda, fracción del río Sinú y Manso. Manifestaciones culturales del pueblo Embera Katío. 2001). La temperatura media de la región es de $24^{\circ} \mathrm{C}$, con precipitaciones anuales entre los 2.000 y $4.000 \mathrm{~mm}$, características que hacen de ella un lugar con una diversidad ecológica especial para el país. La población de indígenas es de 2.500 personas aproximadamente, que viven en 24 comunidades ubicadas a lo largo del río Sinú y sus afluentes, el Esmeralda, el Verde y el Manso, que utilizan como fuente vital de sus necesidades alimentarias, culturales y de transporte. La agricultura de los Embera-Katio ha sido de subsistencia, con cultivos de yuca, plátano, maíz, arroz y frutos de la selva; la principal fuente de proteínas proviene del pescado de río y en segunda instancia de la caza.

Las condiciones de vida en el resguardo han cambiado por la invasión de colonos y la presencia de grupos al margen de la ley (guerrilla y 
paramilitares), con la consiguiente violencia generada por estos actores y la tala indiscriminada de árboles para comercio y sustitución por cultivos ilícitos, que modificó el ecosistema y contribuyó a la disminución de la caza, de los productos de subsistencia y de los cultivos de arroz. A lo anterior se suma la construcción de la represa hidroeléctrica de Urrá, la cual aprovecha las aguas del río Sinú e interrumpe la corriente del río por el embalse, disminuyendo de manera considerable la pesca del "bocachico" (Prochilodus nigricans), fuente principal de proteína animal para estas comunidades, dificultándoles el desplazamiento por el río en embarcaciones rústicas movilizadas con remo, actividad que les permitía la comunicación con otras comunidades y con la cabecera municipal para el desarrollo social y el abastecimiento de víveres. En la actualidad, el transporte se hace en lancha a motor, lo cual implica para ellos un costo adicional.

El objetivo del presente estudio fue determinar el estado nutricional de los niños y niñas de cero a seis años de edad de este resguardo, e identificar algunos de sus factores condicionantes.

\section{Materiales y métodos}

\section{Tipo de estudio y diseño de la muestra}

Se realizó un estudio transversal entre noviembre de 2001 y mayo de 2002. De las 24 comunidades del resguardo Embera-Katio de Tierralta, Córdoba, se seleccionaron 14 por muestreo aleatorio simple y en éstas se eligieron en forma consecutiva 272 niños y niñas de hasta seis años de edad. La muestra cumplió con los siguientes parámetros estadísticos: universo de 309 niños, nivel de confianza de $99 \%$, error de muestreo de $3 \%$ y prevalencia esperada de $50 \%$, valor que se asumió para obtener un mayor tamaño de muestra, ya que se desconocían en la zona datos sobre prevalencia de desnutrición crónica. En 194 hogares $(100 \%$ de los existentes en las 14 comunidades seleccionadas), se diligenció una encuesta que incluyó datos demográficos de los niños y niñas, historia de su lactancia, alimentación, incidencia de infección respiratoria aguda y de enfermedad diarreica aguda, condiciones de la vivienda, saneamiento ambiental y datos sociodemográficos y de paridad de las mujeres. Para obtener la información sobre alimentación se utilizó el método etnográfico basado en la entrevista en el hogar, con la madre o con la persona delegada por la familia. El cuestionario incluyó preguntas sobre los alimentos que consumían todos los miembros de la familia en un día, preparaciones más comunes, alimentos que adquirían semanalmente o tenían disponibles (comprados, por trueque, cosecha, pesca o caza) para la alimentación familiar y los alimentos suministrados a los niños durante el destete. La entrevista se combinó con la observación directa de los víveres en el hogar y de los alimentos servidos durante un día (8).

Además se utilizaron fuentes secundarias de información como registros de consulta y de egresos hospitalarios para conocer las primeras causas de morbilidad en los menores de cinco años. La prevalencia de parásitos intestinales se estimó con base en los resultados del examen coprológico a una muestra por conveniencia de 172 niños, cuyos padres, o un adulto responsable, estuvieron de acuerdo en participar.

Para medir el peso, la estatura (o la longitud) y el perímetro cefálico se utilizaron procedimientos estandarizados (9). Para el peso se utilizó una báscula de colgar marca Salter con capacidad de 30 kilogramos y precisión de 50 gramos. La longitud y la estatura se midieron en infantómetro y estadiómetro portátil, con aproximación al milímetro más cercano. El perímetro cefálico se midió con cinta métrica de fibra de vidrio de 0,5 $\mathrm{cm}$ de ancho y una precisión de $1 \mathrm{~mm}$. Para garantizar la confiabilidad de los datos se realizaron controles de calidad durante las mediciones antropométricas y se validaron los formularios diariamente y al final del trabajo de campo.

En cada hogar, una persona mayor de 18 años respondió la encuesta y recibió un recipiente de plástico sin ningún líquido preservativo ni diluyente para la recolección de la muestra de materia fecal. Se le solicitó que mediante una paleta de madera recolectara una muestra que debía depositar en el recipiente suministrado. La muestra se procesó en forma inmediata; se realizó una descripción macroscópica de las materias fecales y un 
examen directo con solución salina y lugol. La elección de quien suministraba la información se hacía en el hogar en forma espontánea de acuerdo al rol que desempeñaba y a quien estuviera disponible en el tambo.

Los niños y niñas se concentraron en un tambo principal de cada comunidad para la evaluación antropométrica.

La estatura para la edad, el peso para la estatura y el perímetro cefálico se compararon con los valores de referencia del Centro Nacional de Estadísticas de la Salud de los Estados Unidos de Norteamérica recomendados por la Organización Mundial de la Salud (OMS) para comparaciones internacionales $(2,10)$. Los niños con estatura para la edad inferior a una desviación estándar (DE) con relación a la mediana se clasificaron como casos de desnutrición crónica leve; los inferiores a $2 \mathrm{DE}$, como casos de desnutrición crónica moderada y los que estuvieron $3 \mathrm{DE}$ por debajo de la mediana, como desnutrición crónica grave. Igual criterio se utilizó para clasificar el déficit de peso para la estatura (desnutrición aguda leve, moderada y grave) $(2,10)$. El exceso de peso para la estatura se clasificó como sobrepeso cuando se encontró entre 1 y 2 DE por encima de la mediana, como obesidad si era superior en más de $2 \mathrm{DE}$. El perímetro cefálico se comparó con los valores de los percentiles con la siguiente clasificación: inferior al 5 , igual o superior al 5 e inferior al 25; igual o superior al 25 , e inferior al 75, igual o superior al 75 (2). Respecto al uso de los valores de referencia recomendados por la OMS es importante aclarar que son los únicos existentes para este tipo de comparaciones y, además, se ha demostrado que las principales variaciones en el crecimiento físico entre poblaciones se deben a factores ambientales y socioeconómicos, más que a diferencias étnicas o raciales $(11,12)$.

Un equipo técnico conformado por un epidemiólogo, cuatro bacteriólogos y traductores indígenas que laboraban como promotores de salud se encargó de recolectar los datos. El proceso de estandarización de todos los procedimientos fue realizado por el grupo investigador y verificado en la recolección de información y muestras, para lo cual se realizó crítica de campo diariamente.

\section{Análisis de los datos}

Los datos se procesaron y analizaron en los programas Epi Info versión 6.0 (Centers for Disease Control and Prevention, Atlanta, Georgia, United States) y Microsoft Excel (Microsoft Corp., Rendomd, WA). La asociación estadísticamente significativa entre las variables se determinó mediante la prueba de ji al cuadrado con sus correspondientes razones de momios (Odds Ratio-OR) y se aceptó como significación $p<0,05$.

\section{Consideraciones éticas}

El proyecto fue aprobado por el Comité de Investigaciones del Instituto Colombiano de Medicina Tropical. El consentimiento para la realización del estudio fue dado por los gobernadores de las comunidades y los caciques de los cabildos. El consentimiento se dio en forma verbal. Para dar a conocer el estudio se socializó a través de talleres y entrega de material didáctico, o sea una cartilla explicativa sobre el estudio.

\section{Resultados}

\section{Características sociodemográficas}

De los 272 indígenas estudiados, 129 (47,4\%) eran hombres y $143(52,6 \%)$ mujeres, con una edad promedio de 31,9 meses $\pm 20,1$. En los 194 hogares encuestados habitaban 605 mujeres, con edad promedio de 17,3 años; el grupo de mujeres en edad fértil fue de 238 . El promedio de embarazos por mujer fue de 3,9, con un mínimo de uno y un máximo de 15, y por grupos de edad, 3,3 en las menores de 24 años; 4,6 en el grupo de 25 a 29 años; 7,7 en el de 35 a 39 años; 9 en el de 40 a 54 años, y 11,7 en el de 55 a 59 años, es decir, todo el tiempo de capacidad reproductiva de la mujer Embera es exclusivo para reproducirse, lo que se refleja en una tasa de fecundidad de 230 niños vivos por cada 1.000 mujeres en edad fértil. El promedio de personas por hogar fue de 7,1 y el de menores de cinco años, de $1,8 . \mathrm{La}$ tasa de mortalidad infantil fue de 82 por 1.000 nacidos vivos. Los indígenas tienen acceso muy limitado a la educación formal y predomina la tradición oral en lengua Embera. 


\section{Estado nutricional}

La prevalencia de desnutrición crónica moderada y grave fue de $63,6 \%$, de desnutrición aguda leve y moderada, del $8,8 \%$; no se presentaron casos de desnutrición aguda grave; sin embargo, se observó una tendencia al exceso de peso para la estatura en el $18,4 \%$, distribuido en $15,1 \%$ de sobrepeso y $3,3 \%$ de obesidad. La menor prevalencia de desnutrición crónica moderada y grave se presentó en el grupo de cero a cinco meses $(21,4 \%)$ (cuadro 1). Para el análisis de tendencia se utilizó el grupo menor de seis meses como punto de referencia, encontrando que la prevalencia de desnutrición crónica se asoció con el incremento de la edad ( $p<0,00001)$. La prevalencia total de desnutrición crónica (leve, moderada y grave) fue mayor en los hombres que en las mujeres $(89,9 \%$ frente a $80,4 \%$ : $p=0,028)$.

El $74 \%$ de los menores presentaron un perímetro cefálico inferior al percentil 5 de los valores de referencia (203 niños); el 16\% estaba entre el percentil 5 y el 25 ; el $8 \%$, entre el 25 y el 75 y sólo en el $2 \%$ de los casos era igual o superior al 75. Por grupos de edad se presentó un comportamiento muy similar al de la estatura para la edad, situación que se refleja en el análisis de tendencia, en el que se encontró que el porcentaje de niños y niñas con perímetro cefálico menor que el percentil 5 se incrementaba con la edad ( $p$ $<0,05$ ) (cuadro 2), con porcentaje mayor en el grupo de 61 a 71 meses $(93,3 \%)$. El comportamiento por sexo fue semejante.

\section{Alimentación}

La disponibilidad de alimentos de las familias indica que alimentos como la panela, el arroz, el plátano y el maíz son la base de la alimentación de los indígenas estudiados. Los alimentos de mayor consumo en un día fueron: arroz y panela (100\% de las familias), plátano y maíz (80,4\%). De los alimentos fuente de proteína de alto valor biológico, la carne fue consumida por el 52,6\% de las familias, el huevo y los lácteos por $6,7 \%$ y $1 \%$, respectivamente (figura 1 ). Bebidas como el agua de panela y la chicha de maíz o de arroz hacen parte de la alimentación diaria del $100 \%$ de las familias.

En cuanto a la alimentación del niño, la lactancia materna tiene una duración promedio de 13,6 meses y la base esencial de la alimentación durante el destete es el arroz y el maíz (92,8\%), alimentos preparados principalmente en chicha. El $80,9 \%$ de las madres suministra a los niños plátano, el $28,9 \%$, carne, el $4,6 \%$, huevo, el $4,1 \%$, leche y el $1,5 \%$, frutas y verduras.

\section{Características de las viviendas y saneamiento ambiental}

La vivienda Embera es un tambo construido sobre estacones de madera, aproximadamente a dos metros del suelo; para subir a ella se tiene un

Cuadro 1. Estado nutricional de los Embera-Katío de seis y menos años según índice de estatura para la edad, resguardo Embera-Katío, Tierralta, Córdoba, Colombia, 2002.

\begin{tabular}{|c|c|c|c|c|c|c|c|c|c|c|c|}
\hline \multirow{3}{*}{$\begin{array}{l}\text { Edad } \\
\text { (meses) }\end{array}$} & & & \multicolumn{8}{|c|}{ Desnutrición crónica } & \multirow[b]{3}{*}{ Total } \\
\hline & \multicolumn{2}{|c|}{$\begin{array}{l}\text { Estatura } \\
\text { normal }\end{array}$} & \multicolumn{2}{|c|}{ Leve } & \multicolumn{2}{|c|}{ Moderada } & \multicolumn{2}{|c|}{ Grave } & \multicolumn{2}{|c|}{$\begin{array}{c}\text { Total moderada } \\
\text { y grave }\end{array}$} & \\
\hline & n & $\%$ & $\mathbf{n}$ & $\%$ & $\mathbf{n}$ & $\%$ & $\mathbf{n}$ & $\%$ & $\mathbf{n}$ & $\%$ & \\
\hline $0-5$ & 17 & 60,7 & 5 & 17,9 & 4 & 14,3 & 2 & 7,1 & 6 & 21,4 & 28 \\
\hline $6-11$ & 5 & 16,7 & 10 & 33,3 & 13 & 43,3 & 2 & 6,7 & 15 & 50,0 & 30 \\
\hline $12-23$ & 4 & 7,3 & 10 & 18,2 & 20 & 36,4 & 21 & 38,2 & 41 & 74,6 & 55 \\
\hline $24-35$ & 6 & 12,7 & 12 & 25,5 & 11 & 23,4 & 18 & 38,3 & 29 & 61,7 & 47 \\
\hline $36-47$ & 3 & 7,1 & 7 & 16,6 & 16 & 38,1 & 16 & 38,1 & 32 & 76,2 & 42 \\
\hline $48-60$ & 3 & 7,5 & 7 & 17,5 & 17 & 42,5 & 13 & 32,5 & 30 & 75,0 & 40 \\
\hline $61-71$ & 3 & 10,0 & 7 & 23,3 & 7 & 23,3 & 13 & 43,3 & 20 & 66,6 & 30 \\
\hline Total & 41 & 15,1 & 58 & 21,3 & 88 & 32,3 & 85 & 31,3 & 173 & 63,6 & 272 \\
\hline
\end{tabular}

Prueba de ji al cuadrado para tendencia lineal $=19,09 ; p=0,00001$ 
Cuadro 2. Clasificación por percentiles del perímetro cefálico de niños y niñas de seis y menos años según grupo de edad. Resguardo Embera-Katío, Tierralta, Córdoba, Colombia, 2002.

\begin{tabular}{|c|c|c|c|c|c|c|c|c|c|c|}
\hline \multirow{3}{*}{$\begin{array}{l}\text { Edad } \\
\text { (meses) }\end{array}$} & \multicolumn{8}{|c|}{ Clasificación perímetro cefálico } & \multirow[b]{3}{*}{ Total } & \multirow[b]{3}{*}{ OR } \\
\hline & \multicolumn{2}{|c|}{$<\mathrm{P}^{\mathrm{a}} 5$} & \multicolumn{2}{|c|}{$\geq$ P5 $<$ P25 } & \multicolumn{2}{|c|}{$\geq$ P25 < P75 } & \multicolumn{2}{|c|}{$\geq$ P75 } & & \\
\hline & $\mathbf{n}$ & $\%$ & $\mathbf{n}$ & $\%$ & $\mathbf{n}$ & $\%$ & $\mathbf{n}$ & $\%$ & & \\
\hline $0-5$ & 8 & 28,6 & 9 & 32,1 & 9 & 32,1 & 2 & 7,1 & 28 & 1 \\
\hline $6-11$ & 18 & 60,0 & 9 & 30,0 & 2 & 6,7 & 1 & 3,3 & 30 & 3,8 \\
\hline $12-23$ & 40 & 72,7 & 11 & 20,0 & 3 & 5,5 & 1 & 1,8 & 55 & 6,7 \\
\hline 24-35 & 41 & 87,2 & 3 & 6,4 & 3 & 6,4 & 0 & 0,0 & 47 & 17,1 \\
\hline $36-47$ & 34 & 81,0 & 6 & 14,3 & 2 & 4,7 & 0 & 0,0 & 42 & 10,6 \\
\hline $48-60$ & 34 & 85,0 & 5 & 12,5 & 1 & 2,5 & 0 & 0,0 & 40 & 14,2 \\
\hline $61-71$ & 28 & 93,3 & 1 & 3,3 & 1 & 3,3 & 0 & 0,0 & 30 & 35,0 \\
\hline Total & 203 & 74,6 & 44 & 16,2 & 21 & 7,7 & 4 & 1,5 & 272 & \\
\hline
\end{tabular}

a $P$ : percentiles del perímetro cefálico

Prueba de ji al cuadrado para tendencia lineal $=34,1 ; p=0,00000$ (comparación de $<$ P5 con el resto)

madero con muescas que cumple las funciones de escalera. El tambo no tiene paredes y el piso es de madera. El techo está construido de palma, paja o de otro material vegetal. Sobre el piso, en uno de los extremos de la vivienda, se ubica la cocina con fogón de leña y en otro de los costados está el lugar para dormir en esteras y hamacas. Los tambos se ubican en la selva, cerca de los ríos, y agrupados en comunidades pequeñas. Los animales domésticos viven debajo del tambo donde se protegen y se alimentan. Los perros y las gallinas son los más frecuentes ( 83,9 y $75,6 \%$, respectivamente), le siguen los cerdos $(59,4 \%$ ) y otras aves (46,7\%). El 76\% de los hogares arroja la basura a un terreno baldío o al río y el $24 \%$, la queman o la entierran. La eliminación de excretas es a campo abierto o en el río. El río es el eje central de la vida cotidiana del indígena, es allí donde simultáneamente se obtienen el pescado y el agua para el consumo y se realizan actividades como el aseo personal, el lavado de ropa, la eliminación de basuras y excretas, el transporte y la recreación.

\section{Morbilidad infantil}

Durante el mes anterior a la encuesta, el 69,4\% de los niños y niñas menores de cinco años había presentado al menos un episodio de infección respiratoria aguda (IRA) y el 73,7\% había padecido enfermedad diarreica aguda (EDA). La incidencia de IRA fue más alta en los niños y niñas de seis a 23 meses de edad y la de EDA, en los de seis a 35 meses (cuadro 3). En los niños y niñas con infección respiratoria aguda o enfermedad diarreica aguda provenientes de hogares con más de tres menores de cinco años y de hogares con más de siete personas, la prevalencia de desnutrición crónica fue mayor (cuadro 4).

La prevalencia de parasitosis intestinal en los menores de cinco años fue de $78,1 \%$, con $61,0 \%$ para menores de un año y $83,5 \%$ para los de uno a cuatro años $(p<0,000)$. La prevalencia de parásitos patógenos fue de $68,2 \%$, de los cuales los más frecuentes fueron: Ascaris lumbricoides (43,1\%), Entamoeba histolytica-Entamoeba dispar $(25,9 \%)$, Giardia duodenalis $(18,4 \%)$ y Trichuris trichiura (17,2\%). La prevalencia de parásitos no patógenos fue de $35,1 \%$.

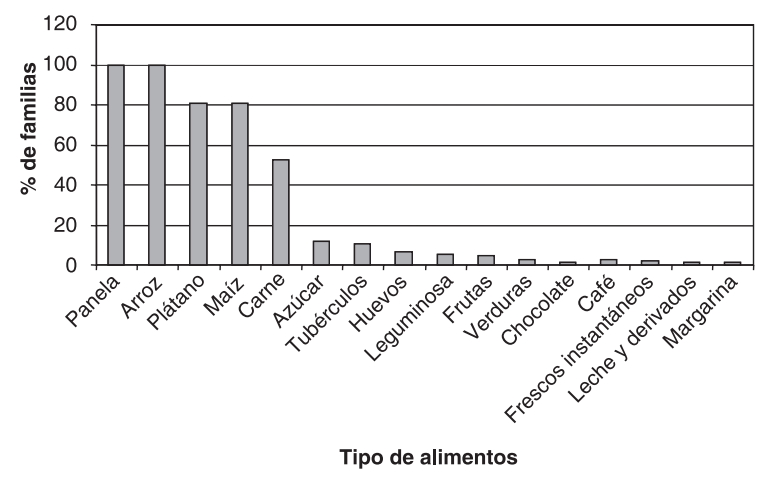

Figura 1. Alimentos consumidos en un día por los EmberaKatío de Tierralta, Córdoba, Colombia, 2002. 
Las cinco primeras causas de consulta externa del grupo menor de cinco años fueron infección respiratoria aguda, enfermedad diarreica aguda, piodermitis, síndrome febril y otitis; las dos primeras representaron el $74,1 \%$. En este grupo de edad, la infección respiratoria aguda, la enfermedad diarreica aguda, el síndrome febril, el paludismo y la desnutrición constituyeron el $87 \%$

Cuadro 3. Incidencia de infección respiratoria aguda y enfermedad diarreica aguda en niños menores de cinco años. Resguardo Embera-Katío, Tierralta, Córdoba, Colombia, 2002.

\begin{tabular}{lccccc}
\hline $\begin{array}{l}\text { Edad } \\
\text { (meses) }\end{array}$ & \multicolumn{2}{c}{$\begin{array}{l}\text { Infección } \\
\text { respiratoria } \\
\text { aguda }\end{array}$} & \multicolumn{2}{c}{$\begin{array}{c}\text { Enfermedad } \\
\text { diarreica } \\
\text { aguda }\end{array}$} & Total \\
& $\mathbf{n}$ & $\%$ & n & $\%$ & \\
\hline $0-5$ & 17 & 73,9 & 16 & 69,6 & 23 \\
$6-11$ & 21 & 77,8 & 24 & 88,9 & 27 \\
$12-23$ & 41 & 78,8 & 39 & 75,0 & 52 \\
$24-35$ & 27 & 61,4 & 35 & 79,5 & 44 \\
$36-47$ & 30 & 71,4 & 29 & 69,0 & 42 \\
$48-60$ & 24 & 61,5 & 27 & 69,2 & 39 \\
$61-71$ & 17 & 60,7 & 18 & 64,3 & 28 \\
Total & 177 & 69,4 & 188 & 73,7 & 255 \\
Pa & & 0,056 & & 0,12 & \\
\hline
\end{tabular}

$\mathrm{P}^{\mathrm{a}}$ : valor de $p$ de prueba de ji al cuadrado para tendencia lineal. de los egresos hospitalarios. Durante el año 2000 se diagnosticaron en el resguardo 392 casos de malaria (260 por Plasmodium vivax y 132 por Plasmodium falciparum). De éstos, 148 (37,7\%) se presentaron en el grupo menor de cinco años. El agente causal en el 73\% (108) de los casos fue Plasmodium vivax y en el $27 \%$ (40) fue Plasmodium falciparum.

\section{Discusión}

Los resultados de este estudio en los niños y niñas Embera Katío de Tierralta, Córdoba, Colombia, muestran una prevalencia de desnutrición crónica moderada y grave del $63,6 \%$, y de desnutrición aguda moderada del 2,2\%. La prevalencia de desnutrición crónica de estos niños es 4,7 veces superior a la del promedio nacional en el año 2000 , que fue de 13,5\%, y la de desnutrición aguda, 2,8 veces más alta (2,2\% de los Embera frente a $0,8 \%$ del promedio nacional) (13). Al comparar los resultados del presente estudio con los realizados en otras comunidades indígenas de Colombia, se observa que la prevalencia de desnutrición crónica moderada y grave supera a la de los Embera del departamento del Cauca (31,1\%); a la de los Wayuu de la Guajira, $(36,9 \%)$; a la de los Arhuacos de la Sierra Nevada $(51,0 \%)$; a la de los Cuna de

Cuadro 4. Factores de riesgo de desnutrición crónica en indígenas de seis y menos años. Resguardo Embera-Katío, Tierralta, Córdoba, Colombia, 2002.

\begin{tabular}{|c|c|c|c|c|c|}
\hline \multirow[b]{2}{*}{ Factor de riesgo } & \multicolumn{5}{|c|}{ Desnutrición crónica } \\
\hline & $\mathbf{n}$ & $\%$ & OR & IC95\% & Valor de $p$ \\
\hline \multicolumn{6}{|c|}{ Infección respiratoria aguda } \\
\hline Sí & 147 & 83,05 & 1,07 & $0,50-2,28$ & 0,84 \\
\hline No & 30 & 82,05 & & & \\
\hline \multicolumn{6}{|c|}{ Enfermedad diarreica aguda } \\
\hline Sí & 158 & 84,04 & 1,39 & $0,64-2,99$ & 0,35 \\
\hline No & 53 & 79,1 & & & \\
\hline \multicolumn{6}{|l|}{ Sexo* } \\
\hline Hombre & 116 & 89,9 & 2,17 & $1,01-4,47$ & 0,028 \\
\hline Mujer & 115 & 80,4 & & & \\
\hline \multicolumn{6}{|c|}{ No. de $<$ de 5 años por hogar } \\
\hline$<3$ & 128 & 80,5 & 0,65 & $0,65-1,37$ & 0,29 \\
\hline$>3$ & 83 & 86,4 & & & \\
\hline \multicolumn{6}{|c|}{ No. de personas por hogar } \\
\hline$<7$ & 127 & 81,9 & 0,86 & $0,42-1,78$ & 0,67 \\
\hline$>7$ & 84 & 84,0 & & & \\
\hline
\end{tabular}

${ }^{*} \mathrm{n}=272$, resto de variables $\mathrm{n}=255$ 
Antioquia $(51,2 \%)$ y a la de los Embera del Chocó $(10,6 \%)(14)$, y que la prevalencia total de desnutrición crónica leve, moderada y grave de los Embera-Katíos de Tierralta (84,9\%) es inferior a la de los Embera de las cuencas de los ríos Ambarradó y Tuguridó (90,5\%) (15) y similar a la de los Embera de Antioquia (83,4\%) (16).

La prevalencia de desnutrición crónica encontrada en este estudio es también superior a la observada en otras comunidades indígenas americanas. En una investigación en indígenas Terénas de Mato Grosso, Brasil, el $16 \%$ de los niños tenían desnutrición crónica (17). En los naporunas de Orellana, Ecuador, la prevalencia fue de $22,8 \%$ (18), en los Panará y Tikunas de Brasil, 12 y 43\%, respectivamente $(19,20)$ y en indígenas de México, $44,3 \%$ (21). La desnutrición aguda fue semejante a la de otros grupos indígenas, a excepción de los pueblos Teréna y naporunas en los que fue superior (5 y $9,8 \%$, respectivamente) $(17,18)$.

La desnutrición crónica de los indígenas EmberaKatío de Tierralta presentó una relación con el incremento de la edad $(p<0,005)$, hallazgo que coincide con el de otros estudios en (15-18,21). La desnutrición crónica es reflejo de las deficiencias acumulativas de alimentación y salud a largo plazo; es un indicador de iniquidad social y refleja las condiciones precarias en que se han criado los niños, por lo cual se asocia directamente con el aumento de la edad. En los Embera se encuentran dos claros incrementos de desnutrición crónica moderada y grave: uno a partir del segundo semestre, en que aumenta un $57,2 \%$ con respecto al primero, y otro en el segundo año, con aumento de 32,9\% con respecto al segundo semestre. Estos dos momentos críticos en el estado nutricional se podrían explicar por el destete y el cambio a una nueva alimentación escasa en proteínas y micronutrientes, además de ser éstas, edades de alta vulnerabilidad a las enfermedades infecciosas. En los niños y niñas mayores se perpetúa una ingestión deficiente de alimentos y enfermedades infecciosas y parasitarias a repetición que continúan deteriorando su crecimiento. Igualmente, el cuidado que se les debe brindar se ve afectado por la llegada de otros hermanos.
La prevalencia de desnutrición crónica en los Embera de Tierralta fue superior en los varones, hallazgo diferente a lo reportado en algunas comunidades indígenas $(16,18)$ y semejante al de los indios Tarahumaras de México (12). Esto puede estar relacionado con procesos culturales, dado que las niñas son más protegidas que los niños por ser consideradas más vulnerables; además, los niños desde pequeños comienzan a desempeñar labores fuera del hogar sin disponibilidad de alimentos durante periodos largos.

Es un hecho conocido que la desnutrición crónica es, en el fondo, el resultado de un proceso de adaptación que se logra a través de una reducción de la velocidad de crecimiento en estatura y que mientras más grave sea la desnutrición y más se prolongue, mayor es su efecto negativo sobre todas las medidas corporales $(22,23)$. Por tanto, el perímetro cefálico inferior al percentil 5 de los valores de referencia es un indicador de desnutrición crónica y se le ha asociado con las condiciones de pobreza, el hacinamiento y la enfermedad diarreica aguda (24). En el presente estudio, el $74 \%$ de los niños presentaron un perímetro cefálico inferior al percentil 5 de los valores de referencia y por grupos de edad presentó un comportamiento muy similar al déficit de estatura para la edad.

La aparente paradoja de alta prevalencia de desnutrición crónica, con adecuación del peso para la estatura y con sobrepeso y obesidad, observada en los Embera es frecuente en comunidades que viven en condiciones de pobreza como resultado del proceso de adaptación a las condiciones ambientales adversas y a una alimentación deficiente en proteínas. Según Guimarey y colaboradores, estas curvas de crecimiento indican que el tipo de alimentación, pobre en proteínas, sobre todo en los primeros años de vida, provoca incremento de la grasa subcutánea, deficiencia de la masa muscular y modificación en las proporciones longitudinales del esqueleto, con acortamiento de los miembros inferiores (25). El patrón dietario practicado por los Embera desde el destete es bajo en alimentos fuente de proteínas de alto valor biológico, así como de minerales y vitaminas. 
La prevalencia de parasitosis fue alta, 78,1\%, cifra que supera la reportada en la Segunda Encuesta Nacional de Morbilidad realizada en Colombia en 1990 (26); además, los niños y niñas mayores de un año tuvieron un riesgo 1,4 veces mayor que los menores de un año. La infección por parásitos es consecuencia de la alta contaminación fecal del ambiente, de la convivencia con animales, de los hábitos higiénicos inadecuados y de la falta de suministro de agua potable, factores que se observaron en esta comunidad. El parasitismo es un proceso negativo para el crecimiento y desarrollo de la población infantil porque aumenta la posibilidad de desnutrición al causar inapetencia, competir por los nutrientes, y provocar diarrea o síndrome de malabsorción. A lo anterior se agrega la alta incidencia de malaria, un factor condicionante de desnutrición infantil. Se conoce bastante el efecto adverso de las infecciones sobre el estado nutricional, cuya gravedad varía con la edad, las prácticas de crianza y el ambiente físico, social y afectivo que rodea al niño. La manifestación física más inmediata de la infección es la pérdida de peso, pero cuando las infecciones se vuelven repetitivas y se prolongan los episodios, disminuye la velocidad de incremento de la estatura y se llega a un franco deterioro del crecimiento (2).

Según el estudio de Chávez y colaboradores en Tezonteopan, una aldea mexicana pobre, la desnutrición se vinculó estrechamente a la salud, el bienestar y las oportunidades educativas de la comunidad y fue tanto la causa como el efecto de las limitadas oportunidades para el desarrollo social y económico de la población (27), situación similar a la de la comunidad Embera, donde los indígenas son pobres, la alimentación es deficiente, hay pocas oportunidades educativas, se carece de infraestructura básica en electricidad, agua potable, eliminación de excretas y basuras y el acceso cultural y geográfico a los servicios de salud y de bienestar familiar es limitado.

En la comunidad Embera-Katío, la tasa bruta de natalidad fue alta, 45,9 nacidos vivos por 1.000 habitantes, el doble de la del país, la cual en el año 2002 se estimó en 22,4 nacidos vivos por 1.000 habitantes (28), y semejante a la de los Embera de Dabeiba (Antioquia) (16), que fue de
48,9 nacidos vivos por mil habitantes. Igualmente, la tasa de fecundidad fue alta, 230 niños nacidos vivos por 1.000 mujeres de 15 a 49 años. En Colombia, en el año 2002 era de 79,5 niños nacidos vivos por 1.000 mujeres en edad reproductiva (28).

Todo lo descrito culmina en un evento final deletéreo, la muerte de los niños y niñas. En el resguardo Embera-Katío la mortalidad infantil fue de 84,7 por 1.000 nacidos vivos, lo que significa que tienen tres veces más riesgo de muerte que el mismo grupo de edad de Colombia, que para el año 2002 fue de 25,9 por 1.000 nacidos vivos (28).

En conclusión, la prevalencia de desnutrición crónica en la población infantil Embera-Katío es elevada y superior a la reportada en otras comunidades indígenas y a la de la población infantil de Colombia. Factores como las enfermedades, la alimentación inadecuada, las prácticas deficientes de crianza, el saneamiento ambiental exiguo, la asistencia en salud casi ausente, la parasitosis y las infecciones son, en última instancia, consecuencia de la pobreza en que viven estos indígenas. La desnutrición de estos niños tiene graves consecuencias que pueden ser de orden biológico y social. Se ha reportado que los niños desnutridos tienen mayor posibilidad de presentar formas graves de infección respiratoria aguda, como lo es la neumonía, por su deficiente respuesta inmunológica (29). La baja talla en las mujeres constituye un alto riesgo obstétrico en su etapa reproductiva, así como de orden social, porque el desarrollo intelectual puede verse afectado en los niños con desnutrición, lo cual, aunado a una baja estatura, limita su acceso laboral. "La desnutrición es una emergencia silenciosa, pero la crisis que desencadena es muy real, y su persistencia tiene unas repercusiones graves y amenazantes sobre la población infantil, la sociedad y el futuro de la humanidad" (30). Los resultados del presente estudio son el punto de partida para el diseño de programas de mejoramiento nutricional y familiar de los indígenas y para evaluar el posterior impacto de las acciones, además de evaluar las que se están realizando en la actualidad y rediseñarlas para mejorar su eficacia y eficiencia, pero desde la concertación comunitaria y familiar. Es 
necesario priorizar en primera instancia a la población de 6 a 12 meses, en donde la transición alimenticia y de cuidados debe hacerse en forma adecuada y fomentar en los adultos un adecuado cuidado de los varones. Además, se debe involucrar un componente de suministro de agua potable, saneamiento ambiental y educación para la prevención de procesos mórbidos relacionados.

Es importante continuar con esta línea de investigación enfocada a evaluar la velocidad de crecimiento de estos niños, comenzando desde el nacimiento para determinar el momento y las causas de este deterioro en el transcurso de los años.

\section{Agradecimientos}

Los autores agradecen al estadístico Horacio Berrío y a Jorge Mario Correa, profesional en sistemas de información en salud, por el procesamiento de los datos. A los gobernadores, caciques, coordinadores y promotores de salud de los cabildos indígenas por su apoyo. Al personal de las diferentes instituciones de salud por la información que facilitaron y a la comunidad indígena por su aceptación del proyecto.

\section{Conflicto de intereses}

Los autores declaran no tener conflicto de intereses.

\section{Financiación}

Este estudio fue auspiciado por el Hospital San José del municipio de Tierralta en el departamento de Córdoba, Colombia.

\section{Referencias}

1. Mata L. The Santa María de Cauqué study: Health and survival of Mayan indians under deprivation, Guatemala. En: Scrimschaw NS, editor. Community-based longitudinal nutrition and health studies: Classical examples from Guatemala, Haiti and Mexico. Boston: International Nutritition Foundation for Develping Countries; 1995. p.29-79.

2. Restrepo MT. Estado Nutricional y Crecimiento Físico. 1a edición. Medellín: Editorial Universidad de Antioquia; 2000.

3. Gopalan C. "Small is healthy?" For the poor not for the rich! En: Gopalan C, editor. Combating undernutrition. Basic issues and practical approaches. Calcuta: Nutrition Foundation of India; Special Publication. Series 3. 1984. p.27-44.
4. OPS. Resolution V of the Meeting of Indigenous Peoples and Health. Winnipeg: OPS; 1993. Document CD37/ 20.

5. Mudarra M. Pobreza y desnutrición, amenaza nacional. Dicen los medios. Revista Siete. Panamá: EPASA; 2000. [Consultado: 27 de septiembre de 2006]. Disponible en: http://www.critica.com.pa/archivo/05092000/ port3.html

6. Tabares E. Ciencia al día AUPEC. Población indígena, en riesgo de desaparecer. Cali: Universidad del Valle; 2004 [Consultado: 24 de septiembre 2006]. Disponible en: http://aupec.univalle.edu.co/informes/mayo97/ boletin37/indigena.html

7. Bordenave S, Picolotti R. Informe sobre derechos humanos y medio ambiente en América. Washington: Centro de Derechos Humanos y Medio Ambiente; 2002 [Consultado: 28 de septiembre 2006]. Disponible en: www.cedha.org.ar/docs/doc93-spa.htm

8. Pelto P, Pelto G. Anthropological research: The structure of inquiry. 2a. edición. Cambridge: Cambridge University Press; 1978.

9. United Nations Department of Technical Cooperation for Development and Statistical Office. How to Weigh and Measure Children: Assessing the Nutritional Status of Young Children in Household Surveys. New York: United Nations; 1986.

10. Organización Mundial de la Salud. El estado físico: uso e interpretación de la antropometría. Ginebra: OMS; 1995. Serie de informes técnicos No. 854.

11. Ulijaszek SJ. Between-population variation in preadolescent growth. Eur J Clin Nutr 1994;48(Suppl. 1):S5-13.

12. Monarrez J, Martínez H. Prevalencia de desnutrición en niños Tarahumaras menores de cinco años en el municipio de Guachochí, Chihuahua. Salud Publica Mex 2000;42:8-16.

13. Ojeda G, Ordóñez N, Ochoa LH. Salud sexual y reproductiva en Colombia. Encuesta Nacional de Demografía y Salud Profamilia 2000. Resultados. 1‥ edición. Bogotá DC: Printex Impresores Ltda; 2000.

14. Tobar LF, Chinchilla M, Mondragón A. Estado alimentario y nutricional de comunidades indígenas y poblaciones afrocolombianas. Terrenos de la Gran Expedición Humana. Serie de Reportes de Investigación. Bogotá: Jaime Bernal Villegas, Pontificia Universidad Javeriana; 1997.

15. Galvez A, Alcaraz GM, Arias MM. Situación de salud materno infantil Embera en el área de posible resguardo de las cuencas de los ríos Ambarradó y Tuguridó. Centro de Investigaciones de Ciencias SocialesCENICS. Facultad de Enfermería. Medellín: Universidad de Antioquia; 1987.

16. Arias MM, Galvez A, Alcaraz GM. Situación de Salud Materno-Infantil en asentamientos Embera-Dabeiba. 
Antioquia. 1985-1986. Investigación y Educación en Enfermería 1988;6:13-33.

17. Ribas DL, Sganzerla A, Zorzatto JR, Tucunduva S. Child health and nutrition in a Teréna indigenous community, Mato Grosso do Sul, Brazil. Cad Saúde Pública 2001;17:323-31.

18. Buitrón D, Hurtig AK, San Sebastián M. Estado nutricional en niños naporunas menores de cinco años en la Amazonía ecuatoriana. Rev Panam Salud Pública 2004;15:151-9.

19. Baruzzi RG, de Barros VL, Rodrigues D, Medeiros de Souza AL, Pagliaro H. Saúde e doença em indios Panará (Kreen-Alalôre) após vinte e cinco anos de contato com o nosso mundo, com ênfase na ocorrência de tuberculose (Brasil Central). Cad Saude Publica 2001;17:407-12.

20. Viera AA, Braga JU, Moraes CL. Health and nutritional conditions of indigenous and non-indigenous riverine children along the rio Solimoes, Amazonas state, Brazil. Cad Saude Publica 2000;16:873.

21. Rivera JA, Monterrubio EA, González-Cossio T, García-Guerra A, Sepúlveda-Amor J. Estado nutricional de los niños indígenas menores de 5 años de edad en México: resultados de una encuesta nacional probabilística. Salud Pública Mex 2003;45 (Suppl. 4):1-11.

22. Ortega MG. Reserva de hierro y estado nutricional en niños derechohabientes de la Clínica Hospital Dr. Miguel Trejo Ochoa, ISSSTE, Colima. [Tesis] Colima: Universidad de Colima; 2001. [Consultado: 27 de septiembre de 2006]. Disponible en: http:// digeset.ucol.mx/tesis_ posgrado/Pdf/Maria\%20 Guadalupe $\% 20 \mathrm{Faustina} \% 200 \mathrm{rtega} \% 20$ Gomez.pdf\#search = \%22Reserva\%20de\%20 hierro $\% 20$ y $\% 20$ estado $\% 20$ nutricional $\% 20$ en $\% 20$ ni\%C3\%B1os\%20 derecho habientes \%20 de\% $201 \mathrm{a} \% 20 \mathrm{Cl} \%$ C3\%ADnica\%20Hospital\%20Dr.\%20 Miguel\%20Trejo\%200choa\%22.

23. Bengoa JM. Panorama mundial de la desnutrición en el siglo XX. Caracas: Sociedad Latinoamericana de
Nutrición-Capitulo Venezolano; 2006. [Consultado: 27 de septiembre de 2006]. Disponible en: http:// www.slan.org.ve/publicaciones/completas/ panorama_mundial_desnutricion_siglo_xx.asp.

24. Sánchez R, Echeverry J, Pardo R. Perímetro braquial y cefálico como indicadores de pobreza y enfermedad diarreica aguda en niños menores de 5 años, Bogotá Rev Salud Pública 2004;6:167-182. [Consultado: 28 de septiembre de 2006]. Disponible en: http://www. scielo. org.co/scielo.php? script $=$ sci_arttext\&pid $=$ S0124 $00642004000200004 \&$ Ing=es\&nrm =iso.\&tlng =es.

25. Guimarey LM, Carnese FR, Pucciarelli HM. La influencia ambiental en el crecimiento humano. Revista de Divulgación Científica y Tecnológica de la Asociación Ciencia Hoy 2006;5. [Consultado: 27 de septiembre de 2006]. Disponible en: http://www.cienciahoy.org.ar/ hoy30/ambiental.htm

26. Botero D, Restrepo M. Parasitosis Humana. $4^{\mathrm{a}}$ edición. Medellín: Corporación para Investigaciones Biológicas; 2004. p.46.

27. Chavez A, Martinez C, Soberanes B. The effect of malnutrition on human development: A 24-year study of well-nourished and malnourished children living in a poor Mexican village. En: Scrimshaw NS, editor. Community-based longitudinal nutrition and health studies: Classical examples from Guatemala, Haiti and Mexico. Boston: International Nutrition Foundation for Developing Countries: 1995.

28. Instituto Nacional de Salud. Situación de Salud en Colombia. Indicadores básicos. Bogota: Ministerio de Salud; 2002.

29. Verger JT, Bradshaw DJ, Henry E, Roberts KE. The pragmatics of feeding the pediatric patient with acute respiratory distress syndrome. Crit Care Nurs Clin North Am 2004;16:431-43.

30. UNICEF. Estado Mundial de la Infancia 1998. [Consultado: 19 de enero 2005]. Disponible en: http:// www.unicef.org/spanish/sowc98sp/. 